\title{
Hematopoietic progenitor cells as possible origins of epithelial thymoma in a human $T$ lymphocyte virus type I $p X$ gene transgenic rat model
}

\author{
Takahiro Tsuchikawa ${ }^{1,2}$, Hitoshi Ikeda ${ }^{1}$, Kazunori Kikuchi ${ }^{1, *}$, Takahiro Tsuji ${ }^{1}$, Tomohisa \\ Baba $^{1}$, Akihiro Ishizu ${ }^{1}$, Yuetsu Tanaka ${ }^{3}$, Hiroyuki Kato ${ }^{2}$ and Takashi Yoshiki ${ }^{1}$ \\ ${ }^{1}$ Department of Pathology/Pathophysiology, Division of Pathophysiological Science; ${ }^{2}$ Department of Surgical \\ Oncology, Division of Cancer Medicine, Hokkaido University Graduate School of Medicine, Sapporo, Japan \\ and ${ }^{3}$ Department of Immunology, Graduate School of Medicine, University of the Ryukyus, Nakahara, Japan
}

\begin{abstract}
We earlier reported that Fischer $344 / j \mathrm{jl}$ strain (F344) rats carrying a unique $p X$ gene of human $\mathrm{T}$ lymphocyte virus type I (HTLV-I) under control of a rat lymphocyte-specific protein tyrosine kinase (p56lck) type I promoter (Ick-pX rats) spontaneously developed epithelial thymomas from the thymic medulla. To investigate the role of bone marrow cells carrying the HTLV-I pX gene in development of thymomas, the bone marrow of normal F344 rats after lethal irradiation was reconstituted by bone marrow mononuclear cells (BMMC) of Ick-pX rats. Epithelial thymomas similar to the original thymoma of Ick-pX rats frequently developed in the nontransgenic recipients within 5 months after the BMMC transplantation. The thymomas expressed the $p X$ gene, thereby indicating the thymoma cells to be of donor BMMC origin. Since the thymoma also developed in nontransgenic recipients reconstituted by BMMC depleted of adherent cells, it is suggested that nonadherent BMMC of donor Ick-pX rats may migrate to and lodge in the thymus of recipient nontransgenic rats then transform into thymoma cells with epithelial characteristics. The thymoma cells were shown to bind to Ulex europaeus Agglutinin-1 (UEA-1) lectin, which binds epithelial cells in the thymic medulla. It was also shown that the nonadherent BMMC fraction used for bone marrow reconstitution contained a number of UEA-1-positive cells. Taken together, UEA1 positive BMMC may be progenitor cells of the epithelial thymoma. The epithelial thymoma in Ick-pX rats sheds light on epithelial cell development in thymic medulla and for oncogenesis of epithelial thymoma in humans. Laboratory Investigation (2004) 84, 245-252, advance online publication, 15 December 2003; doi:10.1038/labinvest.3700028
\end{abstract}

Keywords: epithelial thymoma; bone marrow cell transfer; transgenic rat; HTLV-I pX gene; progenitor cell

Human T lymphocyte virus type I (HTLV-I) is one of the human infectious retroviruses characterized by an etiologic agent of adult T-cell leukemia. ${ }^{1,2}$ HTLV-I infection is also implicated in the pathogenesis of myeloneuropathy ${ }^{3,4}$ and a number of immunological disorders. ${ }^{5}$ Tax protein encoded by a unique $p X$ gene of the HTLV-I genome is a potent transcriptional activator or repressor of several host cellular genes, ${ }^{6,7}$ and functions as a major pathogenetic molecule of

Correspondence: Dr T Yoshiki, Department of Pathology/Pathophysiology, Division of Pathophysiological Science, Hokkaido University Graduate School of Medicine, Kita-15, Nishi-7, Kita$\mathrm{ku}$, Sapporo 060-8638, Japan.

E-mail: path1@med.hokudai.ac.jp

${ }^{*}$ Currently in the Division of Surgical Pathology, Chiba Cancer Center Research Institute, 666-2 Nitona, Chuo-ku, Chiba 2608717, Japan.

Received 3 August 2003; revised 8 October 2003; accepted 20 October 2003; published online 15 December 2003
HTLV-I. To investigate the pathogenetic role of the $p X$ gene, we established several transgenic rat models carrying the HTLV-I $p X$ gene under controls of various promoters. ${ }^{8-10}$ Among them, a transgenic rat carrying the $p X$ gene under control of a rat lymphocyte-specific protein tyrosine kinase (p56lck) type I promoter (lck-pX rat) is a unique rat model for spontaneously occurring epithelial thymomas in the thymic medulla. ${ }^{10}$ The endogenous p56lck type I promoter is specifically active in thymic lymphocytes. However, in our model, mRNA expression of the $p X$ transgene was detected in all tissues tested, because the p56lck type I promoter used ( -269 to +26 ) did not include the region ( -564 to 433$)$, which contains a sequence to potentially lead cellspecific expression. We concluded that this could be a reason to induce the $p X$ transgene expression at significant levels for transformation of thymic medullary epithelial cells. Morphologically, the 
thymoma consists of spindle-shaped epithelial cells and expresses p40Tax as a $p X$ gene product and cytokeratin as an epithelial marker.

In attempts to determine the origin of thymoma cell in lck-pX rats, we transferred bone marrow mononuclear cells (BMMC) of the lck-pX rat into lethally irradiated nontransgenic Fischer 344/jcl strain (F344) rats and determined if same epithelial thymomas as that of lck-pX rats occur in recipient nontransgenic rats. We further studied to see if BMMC contain possible progenitor cells which express the same molecules as the thymoma cells expressed.

\section{Materials and methods}

\section{Animals}

Inbred F344 rats were purchased from Clea Japan (Osaka, Japan). We used the lck-pX transgenic rat line, Tg38 which frequently develop thymoma. ${ }^{10}$ Rats were maintained at the Institute of Animal Experimentation, Hokkaido University Graduate School of Medicine. All animal experiments were done according to the Guide for the Care and Use of Laboratory Animals, Hokkaido University Graduate School of Medicine.

\section{Preparation of BMMC for Reconstitution}

After deep anesthetization with sodium pentobarbital, BMMC were prepared from tibias and femurs of 6-8 weeks old male donor rats. Both ends of the bones were cut, and bone marrow was flushed out with $5 \mathrm{ml}$ of phosphate-buffered saline, using a syringe with a needle. Collected bone marrow cells in the tube were dispersed by shaking the syringe then mixed with an equal volume of density separation solution (Lympholyte-rat, CEDERLANE, Hornby, Canada). After centrifugation at $1500 \mathrm{rpm}$ for $30 \mathrm{~min}$, the mononuclear cell layer at the interface was collected as the total BMMC. To obtain nonadherent (nAd)-BMMC, total BMMC were passed through Sephadex G10 (Pharmacia KK, Tokyo, Japan) columns at $37^{\circ} \mathrm{C}$ to remove adherent cells. Total BMMC were cultured in Petri dishes that contained $\alpha$-modified minimum essential medium containing $10 \%$ fetal bovine serum (FBS) at $37^{\circ} \mathrm{C}$ in $5 \% \mathrm{CO}_{2}$ incubator for $24 \mathrm{~h}$. After the nonadherent cells had been removed, the adherent cells on the Petri dishes were cultured for 14 days with change of fresh medium at 3 day intervals. Adherent cells as Ad-BMMC were collected after digestion with $0.25 \%$ trypsin-EDTA solution for $20 \mathrm{~min}$. Thymuses of all donor rats were histopathologically examined for thymoma.

\section{BMMC Transfer (BMCT) Experiments}

Nontransgenic F344 male rats at age from 5 to 9 weeks of age were used as recipients of BMCT. All recipient rats were given a lethal dose of radiation (12 Gy) before BMCT. About $10^{7}$ cells of unseparated total, nAd- or Ad-BMMC were injected intravenously in each experiment. Three experimental groups were designed. One group was given an intravenous injection of unseparated total BMMC from lck-pX donor rats, another group was given nAd-BMMC from lck-pX rats, and the third group was given Ad-BMMC from lck-pX rats mixed with $10^{7}$ of total BMMC from nontransgenic F344 male rats. As a control group, recipient rats were given total BMMC from nontransgenic F344 male rats. All recipient rats of the experimental groups were histopathologically examined when symptoms indicating development of thymoma occurred, such as thoracic enlargement, tachypnea or dyspnea, were observed, or when rats reached at 45 weeks after the BMCT. The control group was examined at about 20 weeks after the BMCT. In addition, total BMMC of nontransgenic rats were transferred into lck-pX rats for a comparative group, by the same procedure described above. Recipient rats that had died within 2 weeks after the BMCT were discarded from the count as a technical failure.

\section{Separation of Thymocytes and Thymic Adherent Cells}

Thymus or thymoma of rats were removed aseptically and cut into small fragments with scissors in Dulbecco's modified eagle's medium (DMEM) supplemented with $10 \%$ FBS, $5 \times 10^{-5} \mathrm{M}$ 2-mercaptoethanol and $50 \mu \mathrm{g} / \mathrm{ml}$ streptomycin. Two or three pieces of the fragments were put into each well of a 24-well flat-bottomed culture plate and cultivated with $200 \mu \mathrm{l}$ of DMEM with $50 \%$ FBS in a $5 \% \mathrm{CO}_{2}$ incubator at $37^{\circ} \mathrm{C}$. Without discarding the medium, $100 \mu \mathrm{l}$ of DMEM with $10 \%$ FBS was added to the wells at 3-day intervals. After incubation for 2 weeks, nonadherent cells were collected as thymic lymphocytes. Adherent cells were washed with PBS, removed with trypsin-EDTA solution and seeded into $10 \mathrm{~cm}$ Petri dishes with $10 \mathrm{ml}$ of DMEM with $10 \%$ FBS for a subculture. After 2 weeks with changing the medium at 3-day intervals, the adherent cells were collected as thymic adherent cells.

\section{Histopathology and Immunohisto(cyto)chemistry}

Thymomas of nontransgenic F344 rats given nAdBMMC of lck-pX rats were fixed in $10 \%$ phosphatebuffered formaldehyde and embedded in paraffin blocks. Each $4 \mu \mathrm{m}$ of sections was stained with hematoxylin and eosin. For immunohisto(cyto)chemical analysis, mouse monoclonal anti-cytokeratin (MNF116, DAKO, Glostrup, Denmark) and antiTax (Lt-4), ${ }^{11}$ for detecting the product of the $p X$ transgene, antibodies and biotinylated Ulex Europaeus Agglutinin-1 (UEA-1) lectin (Vector Laboratories, Burlingame, CA, USA) were used as first detecting reagents to histological tissue sections of 
thymus and thymoma of nontransgenic F344 rats given nAd-BMMC of lck-pX rats and to BMMC of lck-pX rats fixed on a glass slide. To develop these first reactants, an avidin-biotin immunoperoxidase kit (DAKO) was used. After immunostaining, tissue sections or fixed BMMC were counter-stained with Mayer's hematoxylin (Merck, Darmstadt, Germany).

\section{Karyotype Analysis}

Chromosomes of separated thymoma cells from an original thymoma of lck-pX rats and a thymoma developed in nontransgenic rats with BMCT from lck-pX rats were analyzed, using a standard G-bands by trypsin using Giemsa method. ${ }^{12}$ In all, 17 cells for original thymoma and 10 cells for BMCT thymoma were analyzed.

\section{Polymerase Chain Reaction (PCR) and Reverse Transcriptase (RT)-PCR}

Total DNAs were extracted from either cell pellets of about $1 \times 10^{6}$ of separated thymocytes, thymic adherent cells, or thymoma cells, and PCR amplification was done to detect integration of the $p X$ transgene according to methods described elsewhere. ${ }^{8}$ Expression of the $p X$ transgene was detected using RT-PCR..$^{8,9}$ Briefly, total RNAs were extracted from separated thymoma cells and the thymoma tissues using RNA extraction kits (ISOGEN, Nippon Gene, Toyama, Japan) and treated with DNase, then they were reversely transcribed with RT and the cDNAs were amplified using a pX specific primer pair to detect the transgene and a rat $\beta$-actin primer pair ${ }^{13}$ as an internal control.

\section{Quantitative RT-PCR}

Expression of the winged-helix-nude (whn) gene in the thymoma was quantitatively measured, using real-time RT-PCR. After cutting small pieces of tissues, the lck-pX thymoma, and nontransgenic thymocyte-depleted thymus and spleen as controls, total RNAs were extracted using ISOGEN. After DNase treatment, total RNAs were reversely transcribed with RT and a random hexamer primer mixture. whn gene expression levels in $1 \mathrm{ng}$ of the resulting cDNAs were quantified using a QuantiTect STBR Green PCR kit (Qiagen GmbH, Hilden, Germany) and ABI PRISM 7900 HT Sequence Detection System (Applied Biosystems, Foster City, CA, USA). Quantitative PCR was done with primers $5^{\prime}$-CCCAAGCTTTGGACAATGGT-3' for sense and $5^{\prime}$ CTGATGAAAGGTGGGCTGAGA- $3^{\prime}$ for antisense ${ }^{14}$ (Genbank accession number S80120) according to methods described in the manufacturer's handbook. For standardization, the glyceraldehyde-3-phosphate dehydrogenase (GAPDH) gene, one of the house-keeping genes, was quantified in each sample, using primers 5'-GGGAGTTGCTGTTGAAGTCA- $3^{\prime}$ for sense and 5'-CCGAGGGCCCACTAAAGG$3^{\prime}$ for antisense ${ }^{15}$ (Genbank accession number M17701). Four lck-pX thymomas, three nontransgenic thymuses and one nontransgenic spleen were used and all experiments were done triplicate and average amounts of whn expression were indicated by a ratio against those of GAPDH expression in each sample.

\section{Flow Cytometry}

BMMC were reacted with fluorescence thiocyanateconjugated UEA-1 (Vector Laboratories), then were analyzed using FACScan (Becton Dickinson, Franklin Lakes, NJ, USA).

\section{Results}

Development. of Epithelial Thymomas in Normal F344 Rats Reconstituted by BMMC from lck-pX Rats

To examine the role of the HTLV-I $p X$ gene in BMMC, about $10^{7}$ cells of total BMMC from lck-pX rats were transferred into lethally irradiated nontransgenic F344 rats. By reconstructing bone marrow by lck-pX rats, thymoma developed in 18 of 19 recipient normal rats within 5 months after the BMCT. BMMC from donor rats with or without primary thymoma did not affect the outcome (Table 1 and Figure 1a). The tumor, which had medullary growth, consisted of spindle-shaped cells expressing cytokeratin as an epithelial marker, similar to the original thymomas in lck-pX rats (Figure $1 \mathrm{~b}$ and c). The p40Tax protein expression as an HTLV-I pX gene product was immunohistochemically detected

Table 1 Development of thymomas in normal F344 rats received BMMC from lck-pX rats

\begin{tabular}{llcc}
\hline Combination $^{\mathrm{a}}$ & $\begin{array}{l}\text { Cells } \\
\text { transferred }\end{array}$ & $\begin{array}{c}\text { Thymoma in } \\
\text { donors }^{\mathrm{b}}\end{array}$ & $\begin{array}{c}\text { No. thymoma } \\
\text { no. recipient } \\
\text { rats }\end{array}$ \\
\hline lck-pX to N & Total BMMC & + & $14 / 14$ \\
& Total BMMC & - & $4 / 5$ \\
& nAd-BMMC & + & $2 / 3$ \\
N to lck-pX & Ad-BMMC & + & $0 / 17^{\mathrm{d}}$ \\
N to N & Total BMMC & - & $3 / 3$ \\
& Total BMMC & - & $0 / 5^{\mathrm{e}}$ \\
\hline
\end{tabular}

${ }^{a}$ BMCT experiments were done on lck-pX rats to nontransgenic rats (lck-pX to N) or nontransgenic rats to lck-pX rats (N to lck-pX). As a negative control, BMMC of nontransgenic rats were transferred to nontransgenic rats ( $\mathrm{N}$ to $\mathrm{N}$ ).

${ }^{b}$ Development of thymomas in donor rats was histopathologically examined when BMMC were prepared.

${ }^{\mathrm{c}}$ All thymomas developed in recipient rats were shown to have histopathological features similar to the original thymomas in lck-pX rats.

${ }^{d}$ All recipient rats were histopathologically examined at about 45 weeks after the BMCT.

${ }^{\mathrm{e}}$ No neoplastic change was found in thymuses of all recipients at about 20 weeks after the BMCT. 

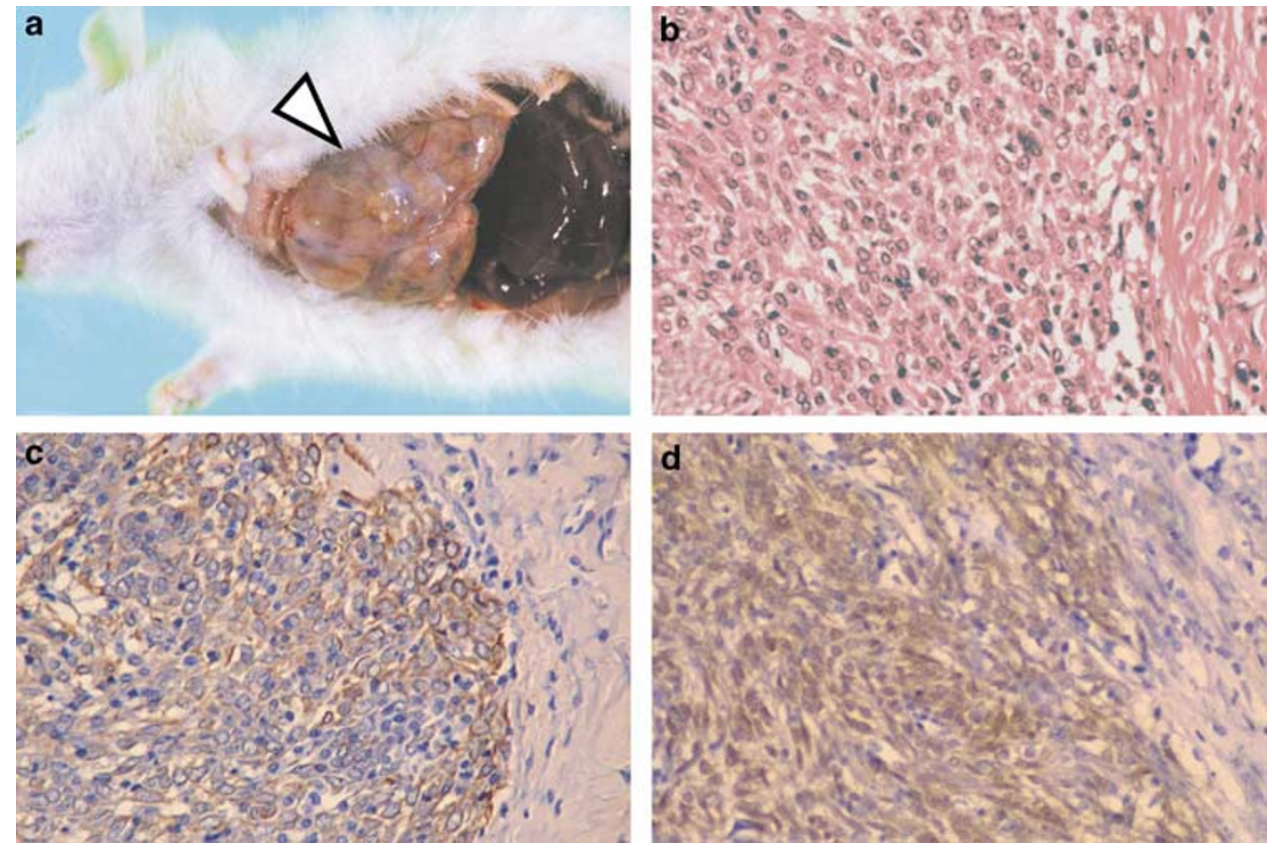

Figure 1 Development of thymomas in normal F344 (nontransgenic recipient) rats after being given total BMMC of lck-pX rats. A large tumor in the anterior mediastinum of a recipient rat at 20 weeks after the BMCT is shown (a arrowhead). Microscopically, the tumor, similar to the lck-pX thymoma, is composed predominantly spindle-shaped cells (b) (HE staining, original magnification: $\times 125$ ). Immunohistochemically, spindle-shaped tumor cells are uniformly stained by anti-cytokeratin (c) and anti-Tax monoclonal antibodies (d) (original magnification: $\times 125$ ).

in the tumor (Figure 1d). Karyotype analysis showed that the same $42 \mathrm{XY}$ karyotype, 20 pairs of autosome and a sex chromosome pair, as the standard karyotype of rat was found in both separated thymoma cells from original thymoma and thymoma developed in BMCT rats (data not shown). The reconstitution experiments using separated Ad- and nAd-BMMC from lck-pX rats showed similar thymoma developed in two of three recipient nontransgenic rats given nAd-BMMC of lck-pX rats within 40 weeks after the BMCT (Table 1), Development of thymoma was never evident macroscopically and histologically in all 17 recipient rats given AdBMMC of lck-pX rats at about 45 weeks after the BMCT. Therefore, the nAd-BMMC fraction of lck-pX donor rats may contain progenitor cells of epithelial thymoma. On the other hand, thymomas developed in all three irradiated lck-pX rats with total BMMC transfer from nontransgenic rats within 4 months after the BMCT (Table 1).

\section{Detection of pX Gene in Thymoma Cells of Recipient Rats}

PCR and RT-PCR methods were used to determine the presence and expression of the $p X$ gene in thymoma cells of recipient rats. After separation of thymic lymphocytes and adherent cells, the $p X$ gene was evident in both lymphocytes and adherent cells in the recipient rats, regardless of thymoma occurrence (Figure 2a). pX mRNA expression was also evident in lymphocyte-depleted adherent thymoma cells of recipient rats (Figure 2b), but it was out of the detection levels in thymic adherent cells in recipient rats before the development of epithelial thymoma (data not shown).

\section{Characteristics of Epithelial Thymoma Developed in the Medulla of lck-pX Rats}

Expression of the whn gene, which is a forkheadwinged helix transcriptional factor (reviewed in Kaufmann and Knöchel ${ }^{16}$ ) the mutation of which disrupts thymus development and normal hair growth, ${ }^{17}$ was examined in the thymoma of lck-pX rats to determine if the thymoma cells have the same characteristics as thymic epithelial cells originating from the third branchial cleft and pharyngeal pouch, using quantitative real-time RT-PCR. Only minimal levels of the whn gene expression were detected in the thymoma tissues compared with that in the normal thymus (Figure 3). Since the thymoma tissues seem to contain resident stromal cells of the thymus, it appears that thymoma cells do not express the whn gene. The thymoma cells were positively stained for UEA-1 (Figure 4), a kind of fucose-binding lectins and is known to specifically bind to epithelial cells in the medulla of the thymus, ${ }^{18}$ indicating that the thymoma of lck-pX rats originates in UEA-1-positive epithelial cells in the thymic medulla. This observation is consistent with histopathological findings of early lesions of 


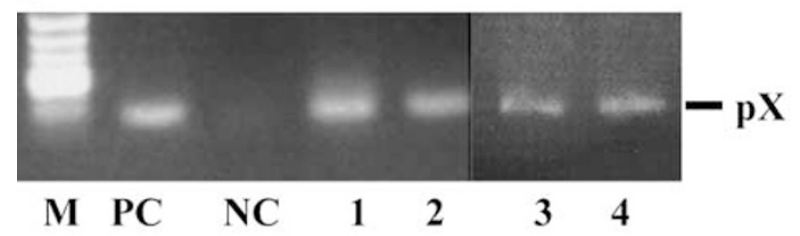

b

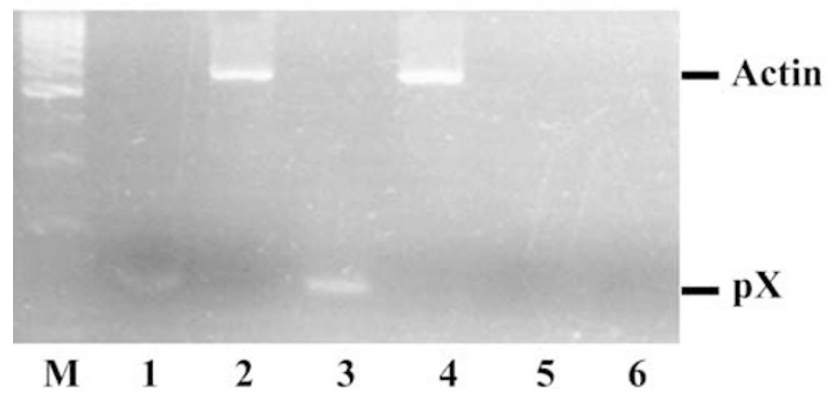

Figure 2 (a) Detection of the $p X$ transgene in thymoma cells isolated from nontransgenic recipient rats received BMMC of lck$\mathrm{pX}$ rats. The $p X$ transgene was detected in both thymic adherent stromal cells (lane 1) and thymocytes (lane 2) isolated from the thymus of a recipient rat before developing thymoma and in both thymoma cells (lane 3) and thymocytes (lane 4) of a recipient rat with a developed thymoma, by PCR. NC indicates thymic adherent stromal cells isolated from an untreated nontransgenic rat as a negative control and PC is thymic adherent stromal cells isolated from an lck-pX rat thymus as a positive control. $\mathrm{M}$ is a Hae III digested $\phi$ X174 DNA size marker. (b) RT-PCR detection of the $\mathrm{pX}$ mRNA in thymoma cells isolated from recipient nontransgenic rats received BMMC of lck-pX rats. Total RNAs were prepared from adherent thymoma cells isolated from a recipient rat (lanes 1,2 and 5) and an original thymoma tissue as a positive control (lanes 3, 4 and 6). Lanes 1 and 3 are results of RTPCR using a pX specific primer pair. Lanes 2 and 4 are using a $\beta$-actin (Actin) primer pair as internal control. Lanes 5 and 6 are results of a pX PCR amplification without RT. M is a $100 \mathrm{bp}$ DNA ladder size marker.

the primary thymoma which apparently originated in the thymic medulla. ${ }^{10}$ Next, we asked if UEA-1positive cells can be observed in BMMC of lck-pX rats, using flow cytometry, even though it is known that megakaryocytes are positive for UEA-1 in humans. ${ }^{19}$ BMMC of the lck-pX rats contained about $7.5 \%$ cells strongly positive for UEA-1 and these were morphologically small mononuclear roundtype cells which differed from megakaryocytes (Figure 5). Cells positive for cytokeratin were not evident in the BMMC fractions (data not shown).

\section{Discussion}

In this study, the transfer of BMMC into irradiated nontransgenic recipient rats from lck-pX rats frequently induced development of thymoma in the host, similar to the original epithelial thymomas spontaneously developed in the lck-pX rats. Since the thymoma cells expressed p40Tax as the $p X$ transgene product, the epithelial thymoma cells in

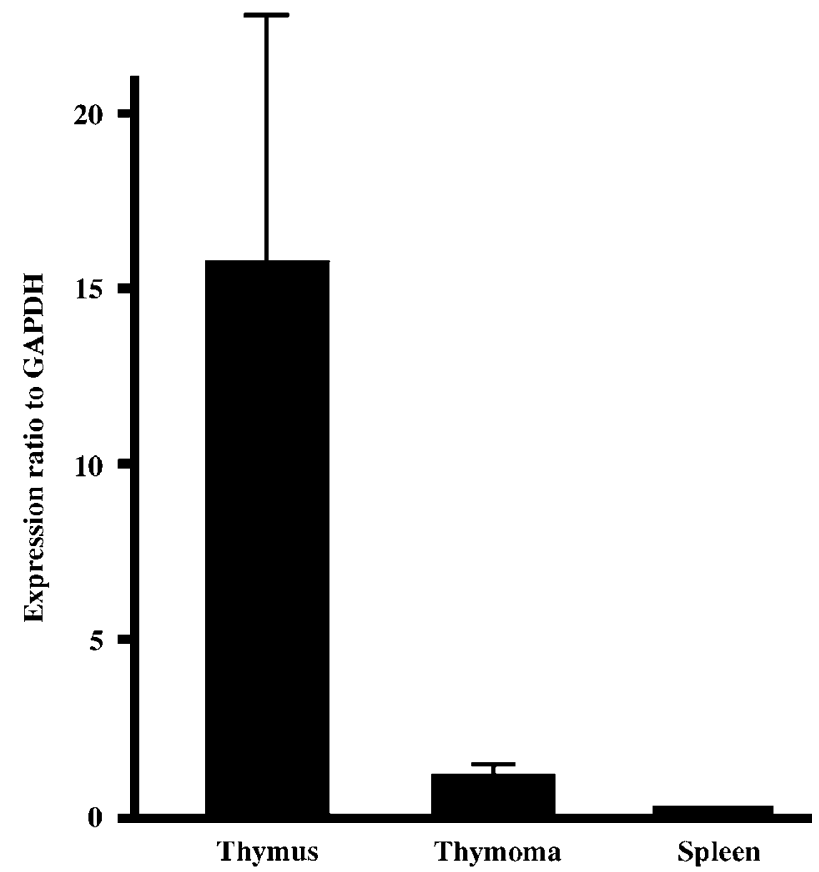

Figure 3 Quantification of whn mRNA expression levels in thymomas. A measure of $1 \mathrm{ng}$ of each cDNA from total RNAs of each tissue was amplified with a whn gene specific primer pair using quantitative real-time PCR. Results are shown as expression ratios against the level of GAPDH expression in each tissue. Thymus means the result of nontransgenic lymphocyte-depleted thymus for a positive control, Thymoma means that of lck-pX original thymomas, and Spleen means that of nontransgenic spleen for a negative control. All experiments were done by triplicate. The average ratio with standard deviation in each experiment group is shown.

the recipient rats would likely have derived from progenitor cells present in the BMMC of donor lckpX rats.

It is generally believed that the epithelial tumor cells in primary thymomas develop from proper epithelial cells which existed constitutively in the cortex or medulla of the thymus, although many bone marrow-derived cells, such as thymocytes and dendritic cells, are principal constituents of thymus. It is generally considered that thymic epithelial cells in the cortex and medulla are derived from ectoderm of the third branchial cleft and endoderm of the third pharyngeal pouch, respectively. The multipotentiality of cells derived from bone marrow, hematopoietic stem cells, was demonstrated in various models, including transdifferentiations into cardiomyocytes, ${ }^{20}$ hepatic cells, ${ }^{21,22}$ existence of precursor cells for bone, cartilage and lung, ${ }^{23}$ and nerve cells. ${ }^{24}$ Donor bone marrow cells could migrate into the recipient thymus and participate in the positive selection of thymocytes as thymic stromal cells by bone marrow transplantation plus bone graft in mice. ${ }^{25}$ In our model, epithelial thymomas with donor characteristics develop in the recipient thymus by $\mathrm{nAd-BMMC}$ transfer, suggesting that nAd-BMMC may contain progenitor 
250
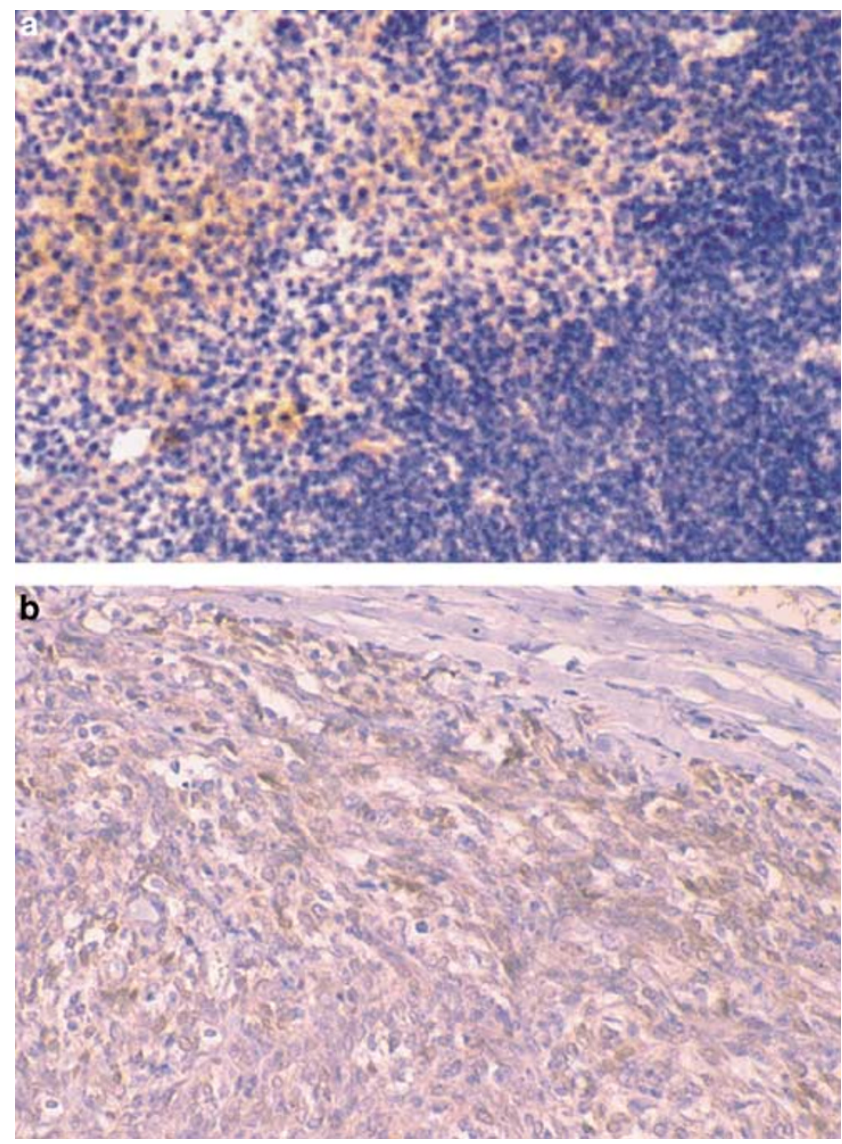

Figure 4 Distribution of the UEA-1-positive cells in the lck-pX rat thymus and the lck-pX rat thymoma. UEA-1-positive cells were localized in the medulla of lck-pX rat thymus before thymoma development (a) (original magnification: $\times 60$ ). Thymoma of lck$\mathrm{pX}$ rat was positive for UEA-1 (b) (original magnification: $\times 75$ ).

cells with the potential to transdifferentiate into a kind of epithelial cells in the thymic medulla. In the thymus, three distinct stromal and epithelial cell types related to T-cell differentiation, cortical and medullary epithelial cells and bone marrow-derived dendritic cells, are known. ${ }^{26}$ Except for dendritic cells, epithelial cells in both areas specifically express not only cytokeratin but also the whn gene a transcriptional factor expressed in epithelial cells of thymic primordium and adult thymus. ${ }^{27}$ whn expression was evident in all the thymomas examined including medullary thymomas in humans. ${ }^{28}$ Therefore, it is reasonable to expect that epithelial thymomas developed from thymic medulla of lck$\mathrm{pX}$ rats would express the whn gene if the thymoma develops from epithelial cells in thymic medulla derived from endoderm of the third pharyngeal pouch. However, expression of the whn gene was minimal in the thymoma of lck-pX rats, which means that the thymoma may not develop from thymic epithelial cells derived from the pharyngeal pouch. It was clearly shown that the epithelial thymomas carrying the $p X$ transgene of lck-pX rats developed in thymus of nontransgenic recipient rats

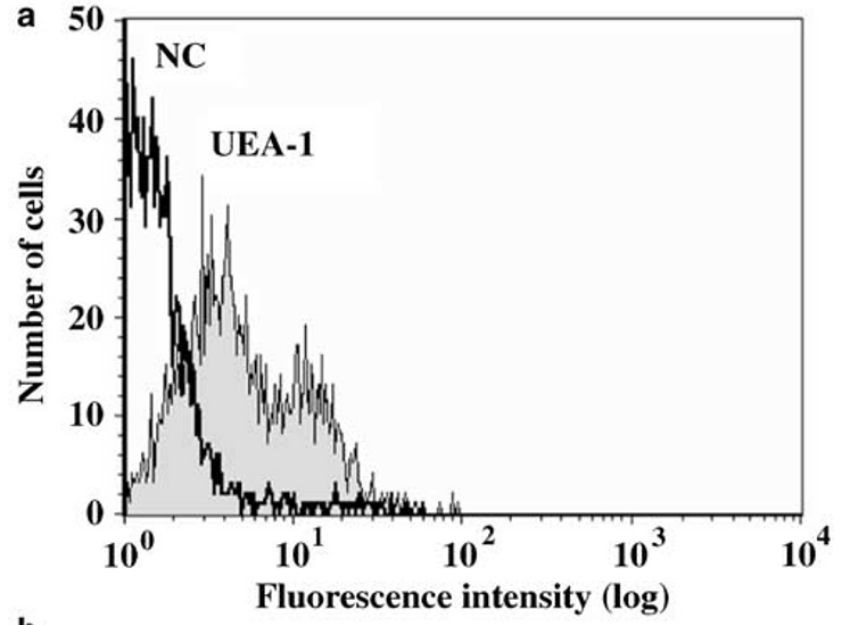

b

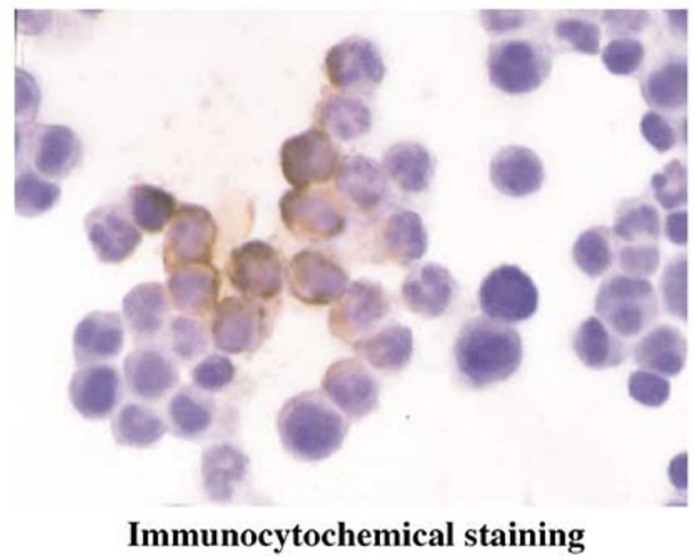

Figure 5 UEA-1 expression of the BMMC in the lck-pX rat. Flow cytometry revealed two peaks of the curve as for the positive intensity of UEA-1 (UEA-1). The population strongly stained with UEA-1 was about $7.5 \%$ of the lck-pX BMMC (a). NC means BMMC without fluorescence thiocyanate-conjugated UEA-1 staining as a negative control. Immunocytochemistry revealed UEA-1-positive cells (brown) to be small round mononuclear ones (b).

by transfer of BMMC from lck-pX rats. The collective evidence indicates that certain medullary epithelial cells of thymus in rats may derive from hematopoietic progenitor cells.

It is also known that there is heterogeneity in epithelial cells of the thymic medulla in rats similar to those in humans and mice. ${ }^{18,29,30}$ UEA-1 high reactivity is only evident in the medulla and can divide epithelial cells of the medulla into UEA-1 positive or negative. We showed that UEA-1-positive cells are clearly localized in the medulla of the rat thymus and the thymomas were positive for UEA-1. About 7.5\% UEA-1-positive cells were evident in nAd-BMMC fraction used for our BMMC transfer experiments, suggesting the possibility that thymomas in the medulla of nontransgenic recipient rats are derived from the transferred BMMC with UEA-1 reactivity, although cells positive for cytokeratin were nil in BMMC fractions. 
Recently, Terada et $a l^{31}$ and Ying et $a l^{32}$ independently suggested that spontaneous cell fusion not transdifferentiation leads to changed phenotypes of neural or bone marrow stem cells to other adoptive cells. However, the possibility of cell fusion in our BMCT model can be negated, since the thymoma developed in BMCT rats had the same morphological and biological features to the original thymoma and no additional chromosome by cell fusion was evident in separated thymoma cells from thymoma developed in BMCT rats.

The thymic organogenesis, including thymic epithelial cells, is still controversial (reviewed in Manley and Blackburn ${ }^{33}$ ). Recent reports suggested that thymic epithelial cells might derive from one progenitor cell in the thymic primordium. Each epithelial islet in the thymic medulla was shown to consist of cells from a single progenitor. ${ }^{34}$ It was also suggested that all functional epithelial cell types of both cortex and medulla might derive from a common progenitor cell with expression of the MTS24 cell surface glycoprotein in the thymic primordium..$^{35}$ Cell fractions containing the progenitor cells were sufficient to fully reconstitute the complex thymic epithelial microenvironment. ${ }^{36}$ However, our results suggest the possibility that bone marrow can supply progenitor cells which migrate and transdifferentiate into epithelial cells in the thymic medulla.

\section{Acknowledgement}

We thank Tsutomu Osanai and the entire staff of the Institute of Animal Experimentation, Hokkaido University Graduate School of Medicine, for maintenance of transgenic rats, Ken-ichi Nakase, Chisato Sudo and Masayo Tateyama for technical assistance and Mariko Ohara (Fukuoka, Japan) for language assistance. This study was supported by grants from the Ministry of Education, Culture, Sports, Science and Technology of Japan.

\section{References}

1 Poiesz BJ, Ruscetti FW, Gazder AF, et al. Detection and isolation of type $C$ retrovirus particles from fresh and cultured lymphocytes of a patient with cutaneous T-cell lymphoma. Proc Natl Acad Sci USA 1980;77: 7415-7419.

2 Yoshida M, Miyoshi I, Hinuma Y. Isolation and characterization of retrovirus from cell lines of human adults T-cell leukemia and its implication in the disease. Proc Natl Acad Sci USA 1982;79:2031-2035.

3 Gessain A, Barin F, Vernant JC, et al. Antibodies to the human $\mathrm{T}$ cell lymphotropic virus type I in patients with tropical spastic paraparesis. Lancet 1985;8452: 407-410.

4 Osame M, Usuku K, Izumo S, et al. HTLV-I associated myelopathy, a new clinical entity. Lancet 1986;p8488: 1031-1032.
5 Hollsberg P, Hafler DA. Pathogenesis of diseases induced by human lymphotropic virus type I infection. N Engl J Med 1993;328:1173-1182.

6 Sodroski JG, Rosen CA, Haseltine WA. A transcriptional protein encoded by the $\mathrm{x}$-lor region of the human $\mathrm{T}$ cell leukemia virus. Science 1984;228: 1430-1434.

7 Fujisawa J, Seiki M, Kiyokawa T, et al. Functional activation of the long terminal repeat of human $\mathrm{T}$ cell leukemia virus type I by a trans-acting factor. Proc Natl Acad Sci USA 1985;82:2277-2281.

8 Yamada S, Ikeda $\mathrm{H}$, Yamazaki $\mathrm{H}$, et al. Cytokineproducing mammary carcinomas in transgenic rats carrying the pX gene of human T-lymphotropic virus type-I. Cancer Res 1995;55:2524-2527.

9 Yamazaki H, Ikeda H, Ishizu A, et al. A wide spectrum of collagen vascular and autoimmune diseases in transgenic rats carrying the env-pX gene of human $\mathrm{T}$ lymphocyte virus type I. Int Immunol 1997;9:339-346.

10 Kikuchi K, Ikeda $\mathrm{H}$, Tsuchikawa $\mathrm{T}$, et al. A novel animal model of thymic tumor: development of epithelial thymoma in transgenic rats carrying human T lymphocyte virus type I pX gene. Int J Exp Pathol 2002;83:247-255.

11 Tanaka Y, Yoshida A, Tazawa H, et al. Production of a recombinant human T-cell leukemia virus type-I trans-activator (tax1) antigen and its utilization for generation of monoclonal antibodies against various epitopes on the tax1 antigen. Int J Cancer 1991;48: 623-630.

12 Wang HC, Fedoroff S. Banding in human chromosomes treated with trypsin. Nat New Biol 1972;235:52-54.

13 Tomaru U, Ikeda $\mathrm{H}$, Ohya $\mathrm{O}$, et al. Human $\mathrm{T}$ lymphocyte virus type I-induced myeloneuropathy in rats: implication of local activation of the $p X$ and tumor necrosis factor- $\alpha$ genes in pathogenesis. J Infect Dis 1996;174:318-323.

14 Segre J, Nemhauser JL, Taylor BA, et al. Positional cloning of the nude locus: genetic, physical, and transcription maps of the region and mutations in the mouse and rat. Genomics 1995;28:549-559.

15 Tso JY, Sun XH, Kao TH, et al. Isolation and characterization of rat and human glyceraldehydes-3phosphate dehydrogenase cDNAs: genomic complexity and molecular evolution of the gene. Nucleic Acids Res 1985;13:2485-2502.

16 Kaufmann E, Knöchel W. Five years on the wings of fork head. Mech Dev 1996;57:3-20.

17 Nehls M, Pfeifer D, Schorpp M, et al. New member of the wing-helix protein family disrupt in mouse and rat nude mutations. Nature 1994;372:103-107.

18 Farr AG, Anderson SK. Epithelial heterogeneity in the murine thymus: fucose-specific lectins bind medullary epithelial cells. J Immunol 1985;134: 2971-2977.

19 Liu SM, Li CY. Immunohistochemical study of Ulex europaeus agglutinin 1 (UEA-1) binding of megakaryocytes in bone marrow biopsy specimens: demonstration of heterogeneity in staining pattern reflecting the stages of differentiation. Hematopathol Mol Hematol 1996;10:99-109.

20 Makino S, Fukuda K, Miyoshi S, et al. Cardiomyocytes can be generated from marrow stromal cells in vitro. J Clin Invest 1999;103:697-705.

21 Petersen BE, Bowen WC, Patrene KD, et al. Bone marrow as a potential source of hepatic oval cells. Science 1999;284:1168-1170. 
22 Lagasse E, Connors H, Dhalimy ML, et al. Purified hematopoietic stem cells can differentiate into hepatocyte in vivo. Nat Med 2000;16:1282-1286.

23 Pereira RF, Halford KW, O’Hara MD, et al. Cultured adherent cells from marrow can serve as long-lasting precursor cells for bone, cartilage, and lung in irradiated mice. Proc Natl Acad Sci USA 1995;92: 4857-4861.

24 Kopen GC, Prockop DJ, Phinney DG. Marrow stromal cells migrate throughout forebrain and cerebellum, and they differentiate into astrocytes after injection into neonatal mouse brains. Proc Natl Acad Sci USA 1999;96:10711-10716.

$25 \mathrm{Li} \mathrm{Y,} \mathrm{Hisha} \mathrm{H,} \mathrm{Inaba} \mathrm{M,} \mathrm{et} \mathrm{al.} \mathrm{Evidence} \mathrm{for} \mathrm{migration} \mathrm{of}$ donor bone marrow stromal cells into recipient thymus after bone marrow transplantation plus bone grafts: a role of stromal cells in positive selection. Exp Hematol 2000;28:950-960.

26 Lo D, Reilly CR, Burkly LC, et al. Thymic stromal cell specialization and the T-cell receptor repertoire. Immunol Res 1997;16:3-14.

27 Nehls M, Kyewski B, Messerle M, et al. Two genetically separable steps in the differentiation of thymic epithelium. Science 1996;272:886-889.

28 Gattenlöhner S, Müller-Hermelink HK, Marx A. Transcription of the nude gene (WHN) in human normal organs and mediastinal and pulmonary tumors. Pathol Res Prac 1999;195:571-574.

29 Waal EJ, Rademakers LH. Heterogeneity of epithelial cells in the rat thymus. Microsc Res Tech 1997;38: 227-236.

30 Such CD, Gao EK, Kosaka H, et al. Two subsets of epithelial cells in the thymic medulla. J Exp Med 1992;176:495-505.

31 Terada N, Hamazaki T, Oka M, et al. Bone marrow cells adopt the phenotype of other cells by spontaneous cell fusion. Nature 2002;416:542-545.

32 Ying QL, Nichois J, Evans EP, et al. Changing potency by spontaneous fusion. Nature 2002;416:545-548.

33 Manley NR, Blackburn CC. A developmental look at thymus organogenesis: where do the non-hematopoietic cells in the thymus come from? Curr Opin Immunol 2003;15:225-232.

34 Rodewarld HR, Paul S, Haller C, et al. Thymus medulla consisting of epithelial islets each derived from a single progenitor. Nature 2001;414:763-768.

35 Bennett AR, Fartey A, Blair NF, et al. Identification and characterization of thymic epithelial progenitor cells. Immunity 2002;16:803-814.

36 Gill J, Malin M, Holländer GA, et al. Generation of a complete thymic microenvironment by MTS24+ thymic epithelial cells. Nat Immunol 2002;3:635-642. 